Supporting information

\title{
Hydrothermal liquefaction of lignin to aromatic chemicals: Impact of lignin structure
}

Yang Cao, ${ }^{a, b}$ Cheng Zhang, ${ }^{c}$ Daniel C.W. Tsang, diajun Fan, ${ }^{e}$ James H. Clark, ${ }^{a, b, e}$ Shicheng Zhanga, $b, f^{*}$

a Shanghai Key Laboratory of Atmospheric Particle Pollution and Prevention (LAP3),

Department of Environmental Science and Engineering, Fudan University, Shanghai 200438, China.

b Shanghai Technical Service Platform for Pollution Control and Resource Utilization of Organic Wastes, Shanghai 200438, China

${ }^{c}$ Zhejiang Province Key Laboratory of Soil Contamination Bioremediation, Zhejiang A \& F University, Hangzhou 311300, China.

d Department of Civil and Environmental Engineering, The Hong Kong Polytechnic University, Hung Hom, Kowloon, Hong Kong, China

e Green Chemistry Centre of Excellence, Department of Chemistry, University of York, York, YO10 5DD, UK 
f Shanghai Institute of Pollution Control and Ecological Security, Shanghai 200092,

China.

Corresponding author: E-mail: zhangsc@fudan.edu.cn 
Table S1. Content of lignin units $(H, G, S)$ in different types of biomass.

\begin{tabular}{lllll}
\hline Biomass & H unit (\%) & G unit (\%) & S unit $(\%)$ & Plant resource \\
\hline Softwood & $0-8$ & $70-80$ & $10-20$ & Spruce, Cypress \\
Hardwood & $0-5$ & $30-50$ & $45-70$ & Birch, Poplar \\
Grass & $10-25$ & $20-50$ & $20-50$ & Corn stover, Rice straw \\
\hline
\end{tabular}


Table S2. Aromatic monomers form reductive/oxidative conversion of lignin.

\begin{tabular}{llll}
\hline Products & $\mathrm{S}, \mathrm{G}, \mathrm{H}$ & Reaction \\
\hline 4-n-propylguaiacol & $\mathrm{G}$ & Reduction \\
4-ethylsyringol & $\mathrm{S}$ & Reduction \\
Phenol & $\mathrm{S}$ & Oxidation \\
Synillin & & & \\
Syringaldehyde & & & \\
\end{tabular}


Table S3. Various lignin models. ${ }^{1-3}$

2-phenoxy-1-phenylethanol


Table S4. Summary of one-pot process and two-step process.

\begin{tabular}{llll}
\hline Entry & Substrate & Advantage & Disadvantage \\
\hline One-pot process & Lignocellulose & High yield; & Low selectivity; \\
& & Simple operation & Complex products \\
Two-step process & Lignin extract & High selectivity; & Modified lignin structure; \\
& & Simple separation & Complex process \\
\hline
\end{tabular}




\section{References:}

1. Deuss, P. J.; Scott, M.; Tran, F.; Westwood, N. J.; de Vries, J. G.; Barta, K. Aromatic monomers by in situ conversion of reactive intermediates in the acid-catalyzed depolymerization of lignin. J. Am. Chem. Soc. 2015, 137 (23), 7456-67.

2. Pelzer, A. W.; Sturgeon, M. R.; Yanez, A. J.; Chupka, G.; O’Brien, M. H.; Katahira, R.; Cortright, R. D.; Woods, L.; Beckham, G. T.; Broadbelt, L. J. Acidolysis of $\alpha-\mathrm{O}-4$ Aryl-Ether Bonds in Lignin Model Compounds: A Modeling and Experimental Study. ACS Sustainable Chem. Eng. 2015, 3 (7), 1339-1347.

3. Shuai, L.; Sitison, J.; Sadula, S.; Ding, J.; Thies, M. C.; Saha, B. Selective C-C Bond Cleavage of Methylene-Linked Lignin Models and Kraft Lignin. ACS Catal. 2018, 8 (7), 6507-6512. 